\title{
ROBOTIC VIDEO-ASSISTED THORACOSCOPIC SURGERY USING CONVENTIONAL THORACOSCOPIC ACCESS
}

Vu Huu Vinh*, Dang Dinh Minh Thanh*, Nguyen Viet Dang Quang*, Truong Cao Nguyen*

\section{SUMMARY}

Video assisted thoracic surgery (VATS) has been widely used and confirmed to be effective and less invasive compared with conventional open surgery. Robotic video-assisted thoracic surgery (R-VATS) is VATSusing a surgeon-controlled robotic system. R-VATS has been increasingly performed worldwide but not in Vietnam. Wehave started implementing r-VATS since July 2018, using conventional thoracoscopic accesses (trocars) and reported our initial results after 18 months of implementation with 116 cases. 57 cases of lobectomy, 9 cases of wedge resection, 19 cases of thymectomy, 28 cases of mediastinal tumour resection, 1 case of esophagectomy, 1 case of oesophageal leiomyoma resection, and 1 case of diaphragm plication. 110 cases had good outcomes with no complications, 5 cases suffered from haemothorax that lasted for more than 5 days. Onepatient died after 35 days due to pneumonia. The operation time was comparable to that ofc- VATS. Average time to chest tube removal was 2 days. Time from surgery to discharge was comparable to that ofc-VATS.

\section{BACKGROUND}

Video-assisted thoracic surgery (VATS) has been proven to be effective and much less invasive than open heart surgery (sternotomy). Its efficacy is also comparable to that of conventional surgery while being less invasive and therefore, VATS is considered superior to open heart surgery. Less invasiveness also means that there is lower risk of complications in high-risk patients such as the elderly and those with comorbid conditions.
Particularly, patients undergoing VATS experience less pain and benefit cosmetically. Taken together, VATS is superior to traditional surgery. Robotic VATS is VATS using a robotic system to assist the surgeon.

Regarding terminologies, VATS stands for videoassisted thoracoscopic surgery, MITS represents minimally invasive thoracic surgery, MIS is minimally invasive surgery and for robot, the commonly used term is robotic-assisted thoracic surgery (RATS). In this paper, we used r-VATS to indicate robotic video-assisted thoracoscopic surgery and wherever VATS is used, it represents conventional video-assisted thoracoscopic surgery or c-VATS.

\section{STUDY METHODS}

The robotic system used in our centre is a $\mathrm{Da}$ Vinci System (U.S.A) with 4 arms. By using 4 arms, we have 5 ports for 5 trocars to introduce 3 surgical equipment (arm 1, 2, 3), one trocar $(12 \mathrm{~mm}$ in the $\mathrm{Si}$ system) for camera and one trocar for human assistant. The assisting port is usually the $12-\mathrm{mm}$ trocar through which the physician assistant could suction or introduce retractor to assist the main surgeon who would be sitting at the control table or put the stapler in. According the manufacturer's recommendations and other surgeons worldwide, all trocars are placed in a linear line in the $8^{\text {th }}$ intercostal space in both left and right sides. The assisting trocar is

\footnotetext{
* Department of Thoracic Surgery, Choray Hospital Scientific director: A/ Prof.|Vu Huu Vinh, PhD

Manuscript received: 01/02/2019-Accepted for publication: 23/03/2020

Scientific reviewer: A/ Prof. Dang Ngoc Hung, PhD Prof. Le Ngoc Thanh, PhD
} 
usually placed in the $9^{\text {th }}$ intercostal space or lungs. At the end of the surgery, one of the trocar below the costal margin (below the $10^{\text {th }}$ rib). All ports is extended to remove the diseased tissue trocars are covered to pump $\mathrm{CO} 2$ with a pressure (Figure $1 \mathrm{~A}$ and $\mathrm{B}$ ). of $8-10 \mathrm{mg}$ into the thoracic cavity to deflate the

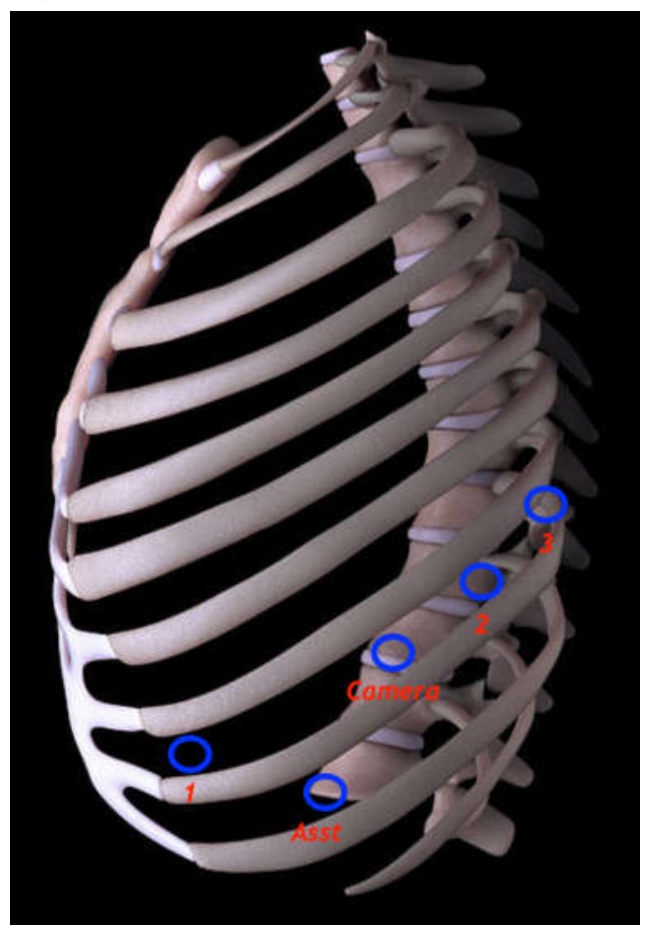

Figure 1A. Linear locations of trocars on the left chest wall. 1, 2, 3: robotic arms and respective equipment. Assit: assisting port, a $12 \mathrm{~mm}$-closed-trocar, the physician assistant uses this port to assist the surgery by providing needle, stitch, gauge, suctioning or introducing the contractor when necessary and this is also the port for stapler introduction to cut vessels, tissue or bronchi-trachea.

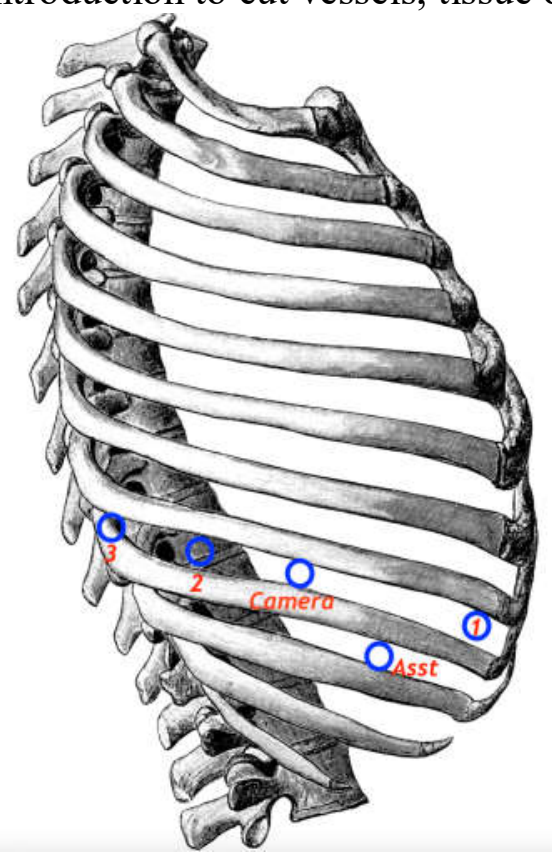

Figure 1B. Linear locations of trocars on the right chest wall 
There are some authors that place the trocars in higher intercostal space $\left(7^{\text {th }}\right)$ but also in a linear line (Figure 1C).

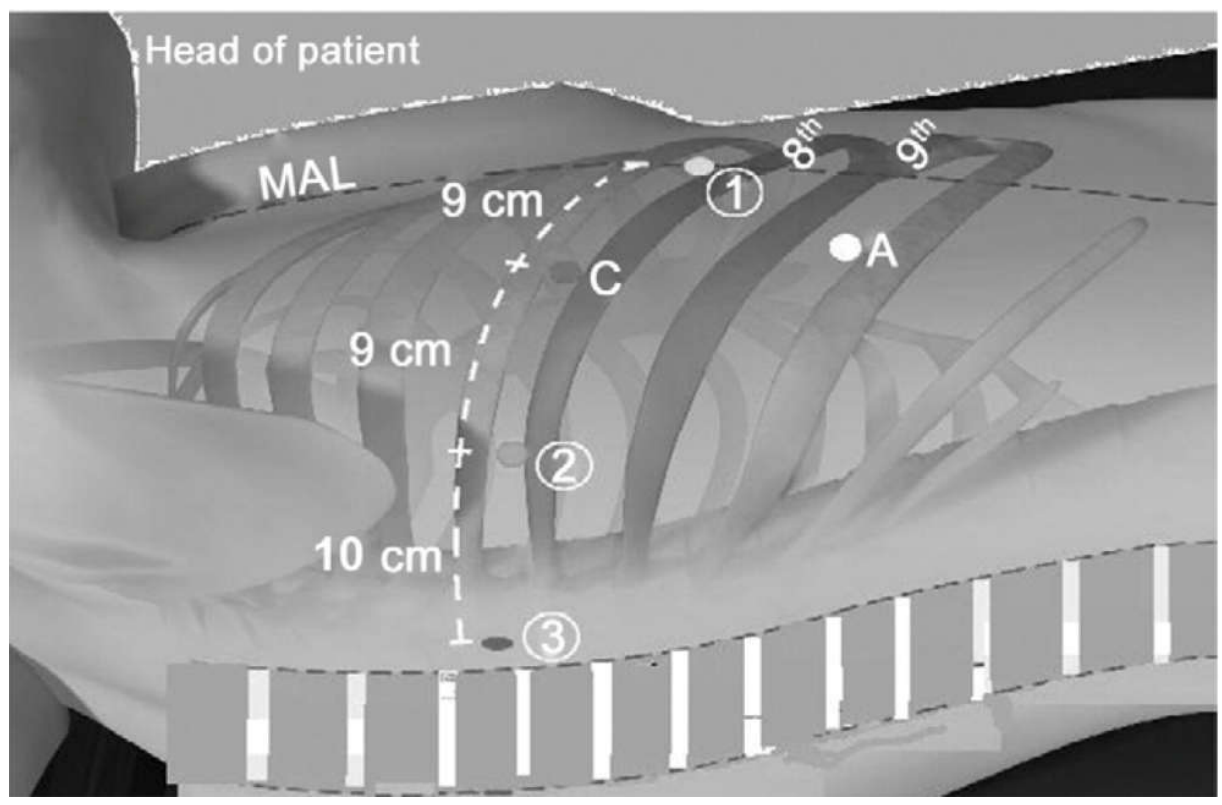

Figure 1C: trocar placed at $7^{\text {th }}$ intercostal space according to Cerfolio (Robert J. Cerfolio, MD, Ayesha S. Bryant, MD, and Douglas J. Minnich, MD. Starting a Robotic Program in General Thoracic Surgery: Why, How, and Lessons Learned. Ann Thorac Surg 2011;91:1729-37)

We only used 4 ports in r-VATS. In surgeries for thoracic diseases such as lobectomy, esophagectomy, oesophageal leiomyoma resection, posterior or one-sided mediastinal tumour removal, we placed the patient on his or her side. The trocars were placed in locations similar to c-VATS following the triangle principle, including 2 trocars for equipment (arm 1 and ), one trocar for camera and one was extended $1.5 \mathrm{~cm}$ from the beginning of the surgery using wound retractor to be the assisting port for physician assistant to suction or introduce the contractor to assist the surgeon during surgery. At the end of the surgery, this $1.5 \mathrm{~cm}-$ port was big enough to take the tissue through without further extension (Figure 2A and B). As we used wound contractor, the thoracic cavity was always open to the room air and $\mathrm{CO} 2$ insufflation could not be used. We deflated the lung required surgery by one-sided ventilation (the opposite side) with double-lumen endotracheal tube with assisting cuff (the third cuff) to navigate the tube precisely inside the desired side (Figure 3). The double-lumen endotracheal tube helped to deflate the lung well, the intubation was simple, effective, and precise. 


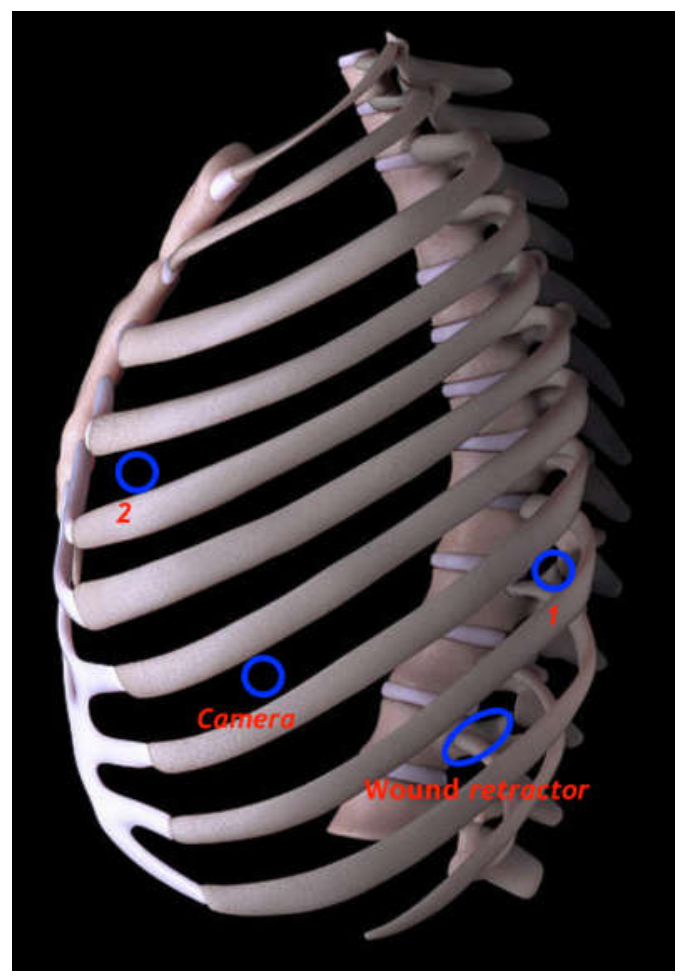

Figure 2A. Locations of trocars in triangle shape following c-VATS for left thoracic surgery. 1,2: robotic arm 1 and 2 (only two arms are used). Wound retractor: the $1.5 \mathrm{~cm}$-port with wound contractor through which the physician assistant could retract when necessary, suction, introduce equipment such as gauge, needle, stitch, etc, this is also the port to introduce stapler to cut vessel, tissue, and bronchitrachea.

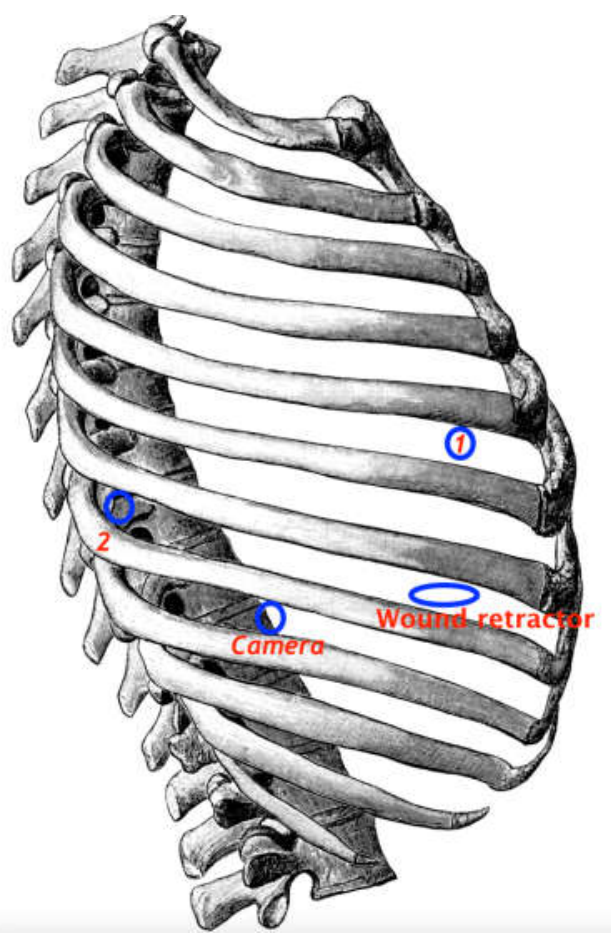

Figure 2B. Locations of trocar in triangle shape following c-VATS for right thoracic surgery 


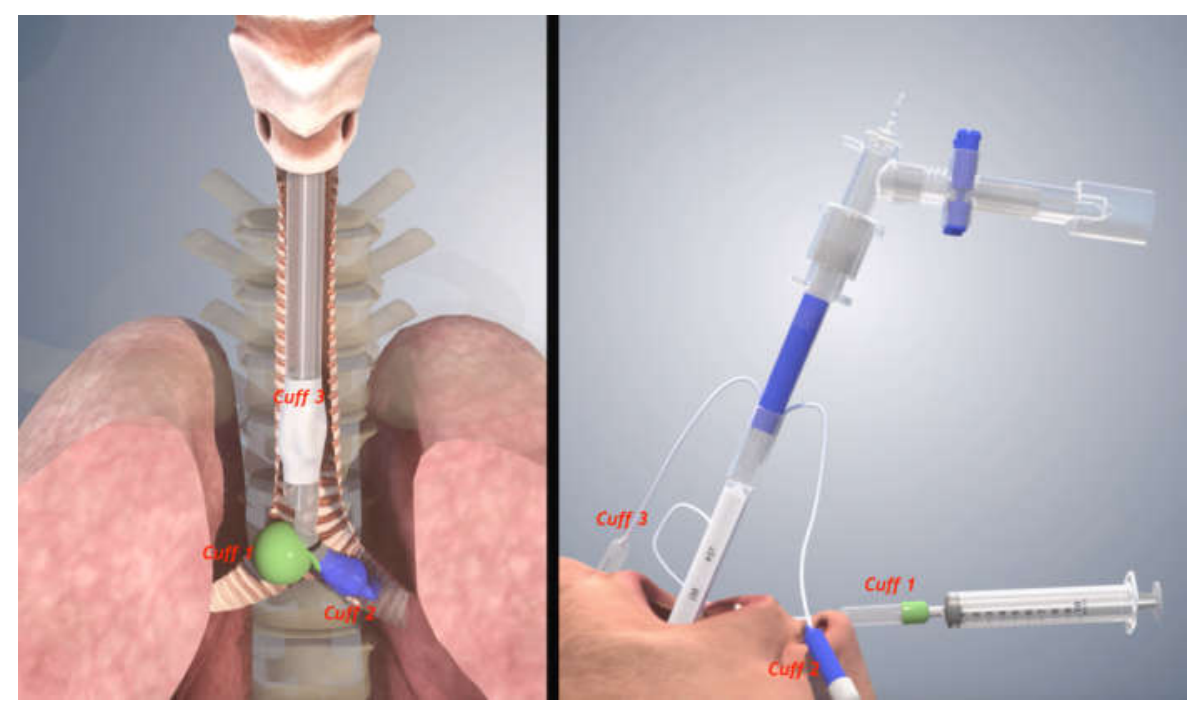

Figure 3: Double-lumen endotracheal tube with 3 cuffs that help to navigate the tube precisely in one branch of bronchi to deflate one side of the lungs easily and effectively.

For surgeries in the anterior mediastinum such as removal of anterior mediastinal tumours, thymectomy for the treatment of myasthenia gravis (regardless of concomitant thymus tumour), we placed the patient in supine position and both lungs were ventilated during surgery but double-lumen endotracheal tube was used in case one-sided ventilation would be necessary. The trocar for camera was placed subxiphoid, two arms 1 and 2 were placed in two sides of the chest, the closed $12 \mathrm{~mm}$ - assistant trocar was placed on either left or right side. The closed system allowed the pump of CO2 to widen and clear the surgical field in the anterior mediastinum without deflating either side of the lungs (Figure 4).

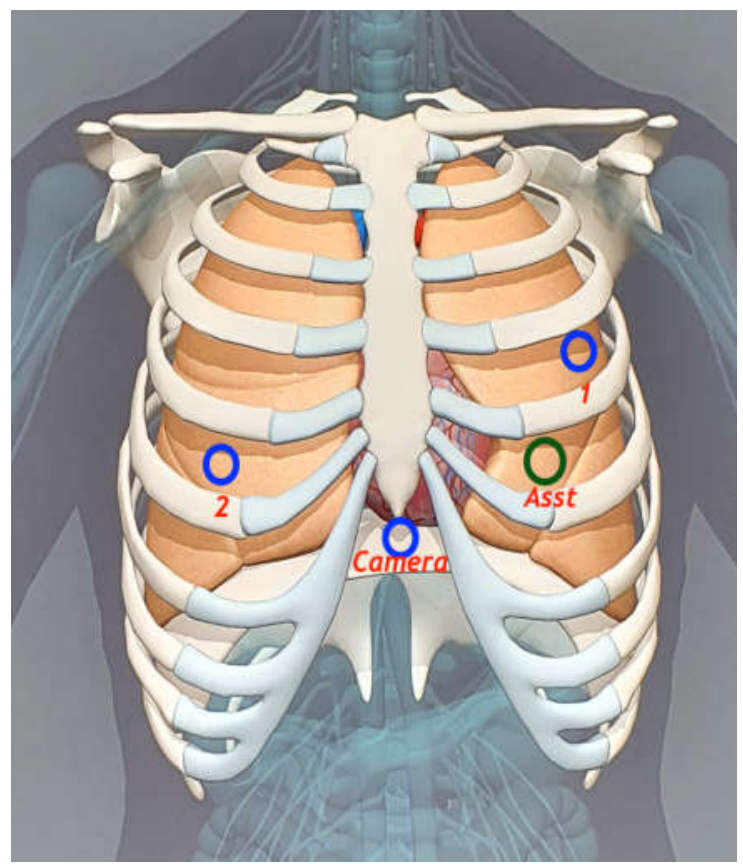

Figure 4: Locations of trocars in surgeries in anterior mediastinum with one sub-xiphoid, CO2 insufflation and two-lungs ventilation during surgery. 1,2: robotic arms 1 and 2; Asst: assistant port, 12 
mm-assistant-trocar, the physician assistant used this port to assist the surgery such as introducing needle, stitch, gauge, suction, retractor, as well as stapler when necessary.

Results: From 7/2018 to 1/2020, 116 patients were operated on including 66 men and 50 women. Specifically, there were 57 cases of lobectomy, 9 cases of wedge resection, 19 cases of thymectomy (regardless of thymus tumour), 28 cases of mediastinal tumours, 1 case of esophagectomy, 1 case of oesophageal leiomyoma resection, and 1 case of diaphragm plication (Table 1).

\begin{tabular}{|l|c|}
\hline Type of surgery & Number of cases \\
\hline Esophagectomy & 01 \\
\hline Oesophageal leiomyoma resection & 1 \\
\hline Lobectomy & 57 \\
\hline Wedge resection & 9 \\
\hline Thymectomy (regardless of thymus tumour) & 19 \\
\hline Removal of mediastinal tumour & 28 \\
\hline Diaphragm plication & 01 \\
\hline Total & $\mathbf{1 1 6}$ \\
\hline
\end{tabular}

Table 1. The number of cases based on types of surgery

There were good outcomes in 110 cases including successful operation, no complications, mean time to drainage removal was 2 days. There were 5 cases that required prolonged drainage and were considered prolonged postoperative haemothorax. In these cases, the mean time to drainage removal was 6 days. After removal the drainage, patients were stable and discharged safely. One patient died due to hospital-acquired pneumonia. This patient was treated for severe pneumonia and died after 1 month. Although no scale was used, all patients reported low level of pain after surgery, quick recovery and satisfaction with r-VATS.

\section{CONCLUSIONS}

Since the 1990s, with the cooperation of NASA and Stanford University, the idea of using robotic surgery in space was proposed with a goal of remotely-controlled surgery. This idea was then commercialised and approved by FDA for civil use. AESOP (Computer Motion, Inc., Goleta, CA, USA) was the first company to use a pedal in combination with a remote control to create the Zeus control system, which was first designed for cardiac surgery and then applied for other surgeries. At the same time, the Da Vinci visual robotic system (Intuitive Surgical, Sunnyvale, CA, USA) with similar inventions also joined the market. In 2003, Da Vinci integrated computerised movements and visual surgery, which became the background for the only robotic surgical system commercially available to date $(1,2)$. In 15 years, the number of robotic surgeries is rapidly evolving in many geographical regions. Thoracic surgeons have successfully applied robotic surgery in their field safely. Robotic surgery has become an option besides c-VATS, all of which improve quality of life more than open heart surgery (3). Over the past 5 years, the introduction of new robotic system such us $\mathrm{Xi}$ in 2014, the operation of 
robotic system has achieved significant advancements. Stapler is now integrated into a robotic arm, camera could be changed in different trocar ports similar to conventional endoscopic surgery. Robotic surgery has remarkable advances to solve challenging vascular cases the factor that could complicate the surgery and is the most common reason for conversion to open surgery in endoscopic surgery (4).

According to the recommendations by the manufacturer as well as many authors, the trocars in robotic surgery should be placed in a linear line in one intercostal space except for the assistant port which is located one or 2 intercostal spaces lower (5). We applied the triangle shape used in conventional thoracoscopy and have shown that this approach was effective and help to reduce on port (only 4 trocars were used instead of 5). This approach allowed better movements of robotic arms due to its direct access to the surgical field. However, due to the principle of robotic arms, the angle formed by the first trocar port, camera and the second arm has to be at least $90^{\circ}$, the distances between trocars must be big enough, at least 4 phalanges (Figure 5) to allow easy movements of robotic arms and not prevent the manipulations of the physician assistant. The use of triangle principle in robotic surgery also helps surgeons to realise the similarities between cVATS and r-VATS in both aspects of surgical field and surgical steps. This approach is also useful for the use of harmonic scalpel with arm 1 (the dominant hand of the surgeon) as this scalpel cannot be folded like other robotic tools. Reducing 1 port also helps to extend other ports to facilitate the surgery. Especially, the physician assistant will be more comfortable in assisting the surgeon and using assisting equipment such as stapler, all of which lead to shortened operation time.

Before our study, there are some authors also placed the trocars in a triangle shape but it was applied on 5 cases of lobectomy only (6). We applied this approach for all robotic surgeries performed in our hospital including lobectomy, esophagectomy, oesophageal leiomyoma resection, removal of mediastinal tumour, thymectomy (regardless of thymus tumour), diaphragm plication, etc.

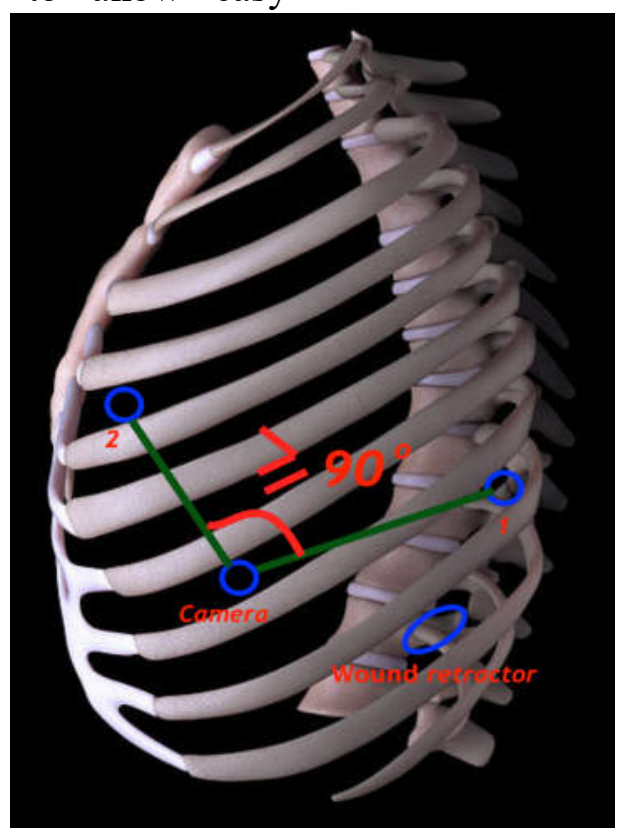

Figure 5: The angle formed by trocar ports number 1 and 2 and camera must be at least $90^{\circ}$. The distances between trocars including wound retractor must be at least 4 phalanges. 
We did not use air pump in lobectomy surgery, instead, we relied on single lung ventilation like thoracoscopy without robotic system and found that the dissection and resection of vessels or bronchi were convenient. With mediastinal tumour or oesophageal, $\mathrm{CO} 2$ pump could be use but with good lung deflation, we did not need additional $\mathrm{CO} 2$ pump. With anterior mediastinal tumour, the camera trocar was placed sub-xiphoid and the patient was placed in supine position, the surgery involved both sides of the chest so pressured pump to extend the cavity under the sternum while ventilating both lungs was necessary. This approach allowed the surgeon to think and operate confidently as if this was an open surgery via sternotomy. In the first cases, we approached from one side of the chest with one-lung-ventilation but we realised that the subxiphoid approach was more convenient so this approach was used since then.

\section{CONCLUSIONS:}

r-VATS was safe, effective, and could be an alternative for c-VATS. The placements of trocars in a triangle shape similar to conventional endoscopic surgery could be applied to robotic surgery. Placing the camera sub-xiphoid was convenient for surgery of the anterior mediastinum such as thymectomy or removal of anterior mediastinal tumour.

\section{REFERENCES}

7. Lane T. A. Short history of robotic surgery. Ann R Coll Surg Engl 2018;100:5-7. 10.1308/rcsann.supp1.5

8. Valero $\boldsymbol{R}$, Ko $\mathbf{Y H}$, Chauhan $\boldsymbol{S}$, et al. Robotic surgery: history and teaching impact. Actas Urol Esp 2011;35:540-5. 10.1016/j.acuro.2011.04.005

9. Kent, M., T. Wang, et al., Open, VideoAssisted Thoracic Surgery, and Robotic Lobectomy: Review of a National Database. Ann Thorac Surg, 2014 Jan; 97(1):236-42.

10. Carmelina C. Zirafa, Gaetano Romano, [...], and Franca Melfi. The evolution of robotic thoracic surgery. Ann Cardiothoracic Surg. 2019 Mar 8(2): 210 - 217

11. Robert J. Cerfolio, MD, Ayesha S. Bryant, MD, and Douglas J. Minnich, MD. Starting a Robotic Program in General Thoracic Surgery: Why, How, and Lessons Learned. Ann Thorac Surg 2011;91:1729-37

12. Bernard J. Park. A complete video-atlas of five robotic-assisted lobectomies. Masters of Cardiothoracic Surgery; Ann Cardiothorac Surg 2012;1(1):100-101. Scan to your mobile device or view video at: www.annalscts.com/article/view/484/580. 